\title{
Esclerose concêntrica de Baló - relato de caso
}

\author{
Balo's concentric sclerosis - \\ case report
}

\author{
Marília Granzotto Volpato', Daniele Gehlen Klaus', \\ Diélly Cunha de Carvalho', Nathalia Tavares Gomes ${ }^{1}$, \\ Rinaldo Claudino²
}

\begin{abstract}
Volpato MG, Klaus DG, Carvalho DC, Gomes NT, Claudino R. Esclerose concêntrica de Baló - relato de caso. Rev Med (São Paulo). 2007 out.-dez.;86(4):207-11.

RESUMO: Objetivos: O caso descrito a seguir busca incentivar a suspeição do médico frente a um provável diagnóstico de Esclerose Concêntrica de Baló (ECB). Métodos: Relato de caso associado à revisão da literatura científica a partir artigos publicados sobre o tema. Resultados: Há grande dificuldade na determinação da etiopatologia da ECB, já que há amplas áreas de desmielinização do sistema nervoso central (SNC) que podem ser observadas em várias doenças neurológicas. A RNM faz grande suspeição diagnóstica, mas a confirmação só é realmente obtida através de biópsia cerebral. $\mathrm{O}$ tratamento consiste no uso de esteróides em doses altas com neuroimagem seriada de controle. Discussão: Uma vez determinada a natureza inflamatória da lesão, a dificuldade de determinar sua etiopatologia reside no fato de que amplas áreas de desmielinização do sistema nervoso central (SNC) podem ser observadas em várias doenças neurológicas. Clinicamente os pacientes com esclerose concêntrica de Balo se apresentam com sintomas agudos ou subagudos, sugerindo lesão expansiva encefálica. A doença tem um curso imprevisível, que varia de uma rápida evolução em semanas ou meses sem remissão até uma condição autolimitada que incluem vários sintomas cognitivos como cefaléia, afasia, distúrbios de comportamento, disfunção visual e ataxia.
\end{abstract}

DESCRITORES: Esclerose múltipla/etiologia. Esclerose cerebral difusa de Schilder/ diagnóstico. Relatos de casos [tipo de publicação].

\footnotetext{
1. Acadêmicas do $5^{\circ}$ ano de Medicina da Universidade do Sul de Santa Catarina - UNISUL.

2. Médico Especialista em Neurologia.

Endereço para correspondência: Marília Granzotto Volpato. Rua Piedade, 256/504. Centro. CEP: 88701200. Tubarão, SC.
} 


\section{APRESENTAÇÃO DO CASO CLÍNICO}

C AS, feminino, 52 anos, refere que há cerca de dois anos iniciou um quadro de incoordenação motora durante a realização de movimentos finos com a mão direita. Posteriormente relatou apraxia em hemicorpo direito. Cerca de quatro dias depois apresentou prejuízo substancial de grafia. Seguiu-se a isso uma fraqueza proximal em dimídio direito, com dificuldade de flexão ao nível da articulação coxo-femoral direita. Também descreveu uma limitação em estender a perna posteriormente além do eixo axial do corpo. Relata fadiga e sensação de fraqueza durante a evolução do caso. Sofreu um episódio de queda de uma escada porque ao descer não conseguiu pôr a perna direita à frente da esquerda.

Nessa ocasião procurou auxílio médico também em virtude de um extenso hematoma na nádega decorrente da queda.

$\mathrm{Na}$ época solicitou-se uma TC e foram estabelecidas três hipóteses diagnósticas: neurocisticercose, tumor cerebral ou infecção cerebral.

O diagnóstico definitivo foi então estabelecido pela ressonância magnética e o paciente encaminhado para a pulsoterapia com corticóide (solumedrona) por 12 dias. Nesse período observou-se hiporreflexia, lentificação dos reflexos e do tempo de planejamento e execução da atividade.

Com o tratamento houve uma melhora progressiva da deambulação e da disgrafia.

Atualmente faz uso avonex (terapia biológica) e fisioterapia.

O único sintoma ainda persistente é a fadiga que ocorre após tarefas repetitivas.

\section{INTRODUÇÃO}

A esclerose concêntrica de Baló (ECB), também conhecida por esclerose difusa mielinoclástica, doença de Schilder, esclerose transicional ou desmielinização pseudotumoral, é uma doença desmielinizante de caráter auto-imune sem causa conhecida do sistema nervoso central, sem etiologia conhecida, mas provavelmente se trata de uma variante rara da esclerose múltipla e foi descrita inicialmente por Jószef Baló em $1927^{1,2}$. Na esclerose múltipla (EM), entretanto, as placas de desmielinização estão bem delimitadas, afetando o SNC com preferência por certas áreas como a substância branca periventricular, o sistema óptico, cerebelo, tronco encefálico e medula espinhal ${ }^{3}$. Essas placas, tipicamente, não produzem efeito de massa, e esse sinal tem sido utilizado para diferenciar os processos desmielinizantes das afecções tumorais ${ }^{3}$. Apesar disso, não devemos esquecer a possível coexistência de EM e glioma cerebral ${ }^{3}$.

Dados epidemiológicos sugerem que a ECB tem uma prevalência de três casos por um milhão de habitantes por ano e que ocorre mais no sexo feminino ${ }^{4}$. Os casos de esclerose múltipla pseudotumoral não são freqüentes; há pesquisas que apontam para quatro formas pseudotumorais em cada 100 pacientes com esclerose múltipla ${ }^{5}$. Algumas alterações são de comportamento, de comunicação, mutismo, apatia e cefaléia. Clinicamente os pacientes com esclerose concêntrica de Baló se apresentam com sintomas agudos ou subagudos, sugerindo lesão expansiva encefálica ${ }^{6}$. De forma que ECB faz diagnóstico diferencial com esclerose múltipla propriamente dita, neoplasias e infecções ${ }^{2}$. Entretanto, o padrão típico de lesão concêntrica na substância branca é altamente sugestivo de $\mathrm{ECB}^{2}$.

Uma vez determinada a natureza inflamatória da lesão, a dificuldade de determinação da etiopatologia dessa doença reside no fato de que amplas áreas de desmielinização do sistema nervoso central (SNC) podem ser observadas em várias doenças neurológicas ${ }^{4}$.

O tratamento se apóia por essa interface com a esclerose múltipla no uso de esteróides em doses altas com neuroimagem seriada de controle ${ }^{3,7}$.

O objetivo desse estudo é tentar incentivar a suspeição do médico frente a um provável diagnóstico de Esclerose Concêntrica de Baló.

\section{MATERIAL E MÉTODOS}

Revisão de artigos científicos publicados sobre esclerose concêntrica de Baló.

\section{RESULTADOS}

Um estudo realizado recentemente no Brain Medical Center em Viena analisou o tecido de autópsia de 14 pacientes portadores de esclerose múltipla com lesões do tipo Balló, em padrão de lesão tecidual associado à desmielinização neuronal ${ }^{8}$. Esse estudo encontrou modelos de desmielinização que guardam semelhanças com lesões teciduais do tipo hipóxia, associado a uma alta expressão de óxido nítrico sintase em macrófagos e células da micróglia ${ }^{8}$. Além disso, nas áreas de lesão ativa e na região mais externa de mielina preservada, foram encontradas proteínas envoltas em tecido, tais como fator indutor de hipóxia alfa-1 e proteína 70 do infarto agudo do miocárdio expressas somente em oligodendrócitos e em menor grau em astrócitos e macrófagos ${ }^{8}$. Devido a 
seus efeitos neuroprotetores, a região periférica das placas de tecido, onde essas proteínas foram encontradas podem ser resistentes a um dano adicional em uma expansão da lesão e podem, além disso, lembrar uma camada de tecido mielinizado? .

Há relato de caso de ECB em que a imagem obtida por TI-201 SPECT é imprecisa quanto à etiologia, ou seja, o foco de esclerose múltipla não pôde ser distinguido de um tumor cerebral maligno ${ }^{3,9}$. Esse fato sugere que em algumas situações, os exames de imagem podem não ser fidedignos em relação ao diagnóstico, precisando-se assim de biópsia para haver o esclarecimento total do caso ${ }^{3}$.

Observa-se que o tratamento com corticoterapia geralmente provoca recidivas e não induz a remissão total dos focos de desmielinização e, inclusive, muitas vezes a resposta a essas medicações pode ser inexistente ${ }^{4}$, o que não ocorreu com a paciente deste relato, que obteve total regressão das lesões cerebrais causadas pela ECB até este momento.

Percebe-se que esse caso estabelece acordo com a literatura pesquisada, por apresentar clínica e imagética compatíveis. $\mathrm{O}$ advento da $\mathrm{RM}$ tem forte influência no diagnóstico precoce e altamente sugestivo da ECB, e possibilita que o tratamento seja instituído rapidamente, antes que as manifestações atinjam de forma incapacitante a vida dos pacientes ${ }^{2,4}$.

\section{DISCUSSÃO}

A doença apresenta evolução rapidamente progressiva, mas nem sempre fatal principalmente quando precocemente diagnosticada ${ }^{1}$.

No passado, o diagnóstico de ECB era feito somente por achados de autópsia ${ }^{2,10}$. Gonçalves et al. ${ }^{2}$ relatam que o diagnóstico era realizado antigamente post mortem ou por biópsia in vivo.

A ECB possui características de imagem típicas, com anéis concêntricos alternados por áreas de desmielinização e re-mielinização ou mielinização normal. Esse aspecto em camadas interacopladas encontrada na ressonância magnética é altamente sugestivo de Baló4.

A RNM demonstra presença de uma lesão expansiva, intra-axial, localizada na região frontal à esquerda, envolvendo o giro frontal superior, que apresenta hipersinal em T2 e Flair e hipossinal em T1, com impregnação periférica e concêntrica após a injeção de contraste paramagnético. Isto está de acordo com Gonçalves et al, que relatam que os achados de zonas concêntricas de hipersinal nas imagens por RM ponderadas em T2, sugerem fortemente o diagnóstico ${ }^{2}$. Essa lesão mede cerca de $3,0 \times 2,0 \mathrm{~cm}$ nos seus maiores eixos, e apresenta área central que tem tendência à cavitação. Não há, entretanto, edema perilesional ou efeito expansivo significativo sobre estruturas adjacentes (Figura 1). Observamos ainda outras pequenas lesões da substância branca subcortical, que também apresentam hipersinal em T2 e Flair, a maior delas peri-trigonal à direita (Figura 2).

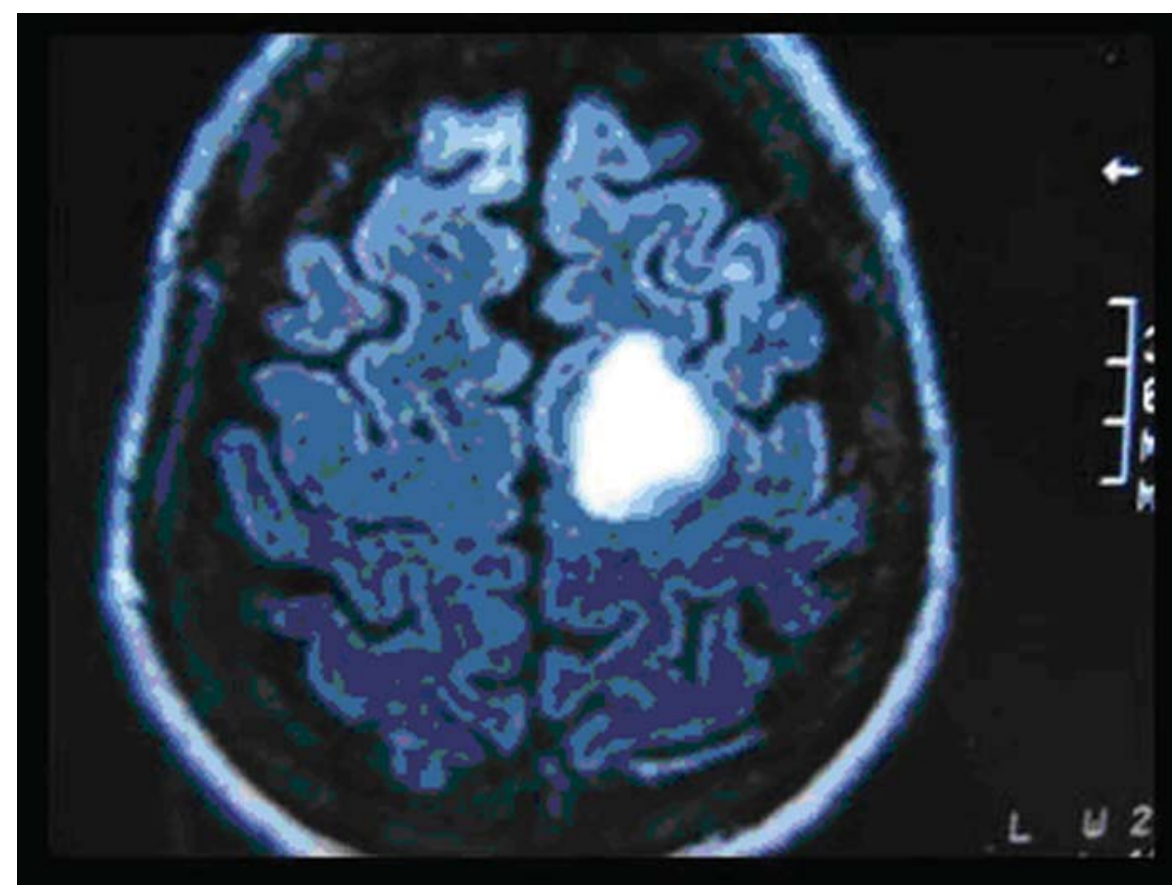

Figura 1. Pequenas lesões na substância branca subcortical (hipersinal em T2 e Flair), sendo a maior delas peri-trigonal à direita 


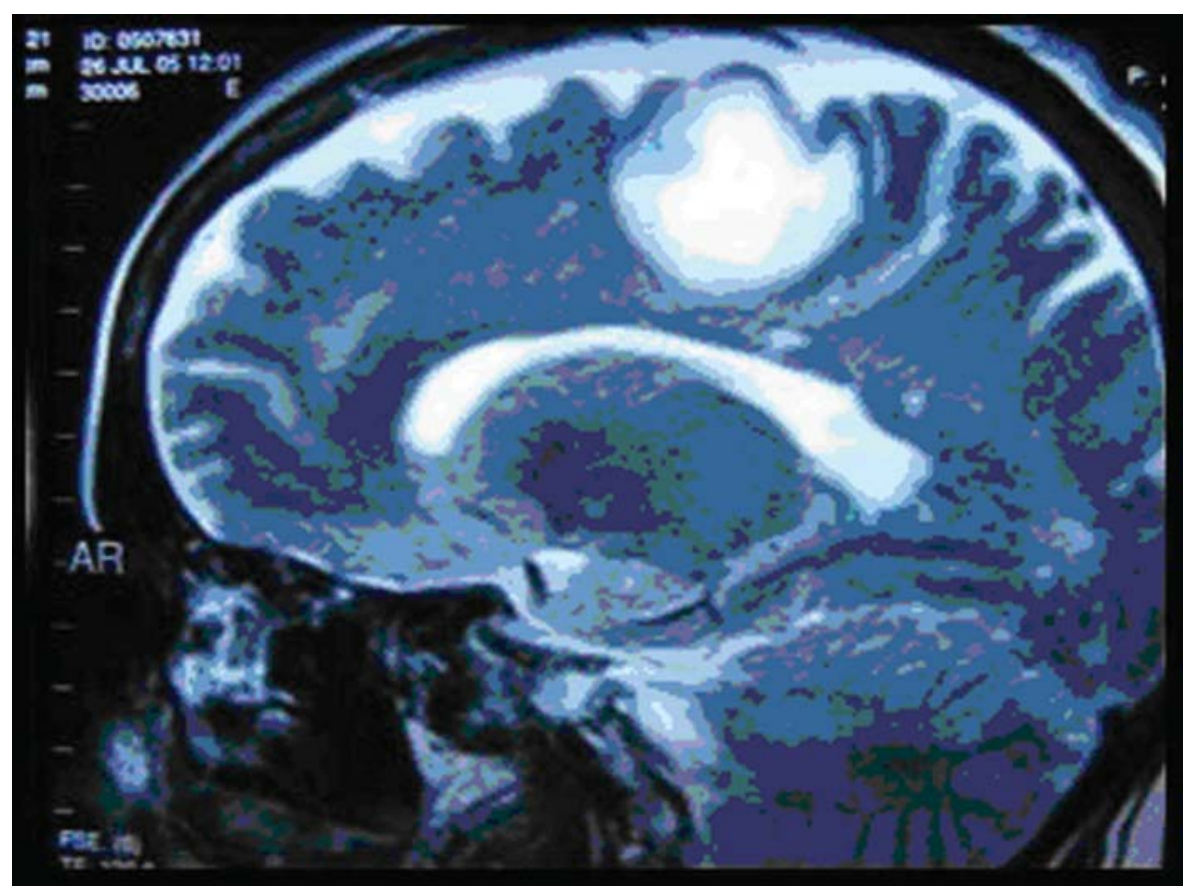

Figura 2. Pequenas lesões na substância branca subcortical (hipersinal em T2 e Flair), sendo a maior delas peri-trigonal à direita

Depois de feito o diagnóstico da doença desmielinizante e descartadas as hipóteses de lesão expansiva intracerebral, acidente vascular encefálico e esclerose múltipla, foi instituído tratamento pulsoterápico com altas doses de corticóides por 14 dias. Salvo algumas intercorrências decorrentes da imunossupressão, a paciente obteve melhora clínica rápida e atualmente tem como queixa apenas fadiga que ocorre diante de atividades prolongadas e repetitivas.

Houve acompanhamento para controle evolutivo através da ressonância após o tratamento com corticóide, o qual e evidenciou regressão da lesão expansiva principal e dos demais focos (Figura 3).

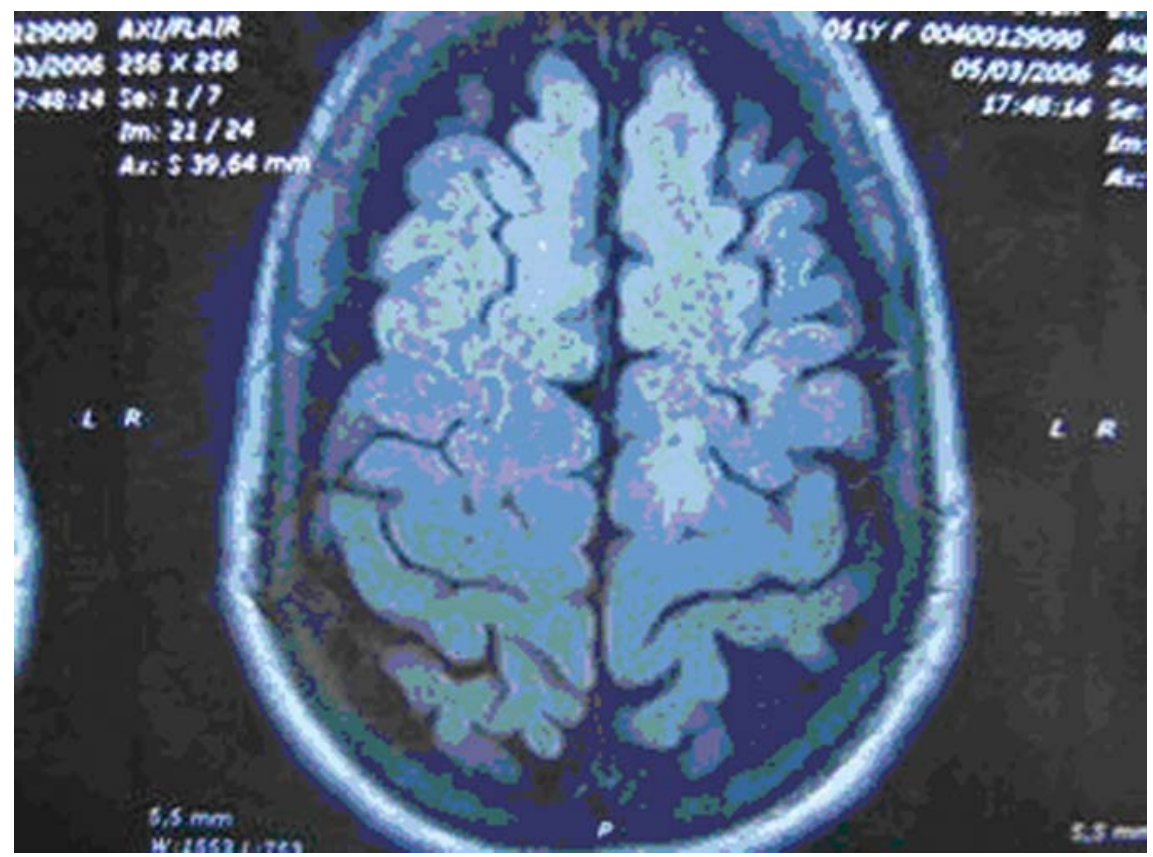

Figura 3. Após tratamento, observa-se regressão da lesão expansiva principal e dos demais focos 


\section{CONCLUSÃO}

Os tratamentos disponíveis para a ECB mostram benefícios sobre a doença e as lesões à ressonância magnética, sendo os principais: metilprednisolona, prednisona, ciclofosfamida e interferon $\mathrm{B}^{6}$. Houve êxito na remissão da crise com a instituição da pulsoterapia com metilprednisolona (solumedron). A paciente se mantém sem reagudização da doença há um ano com o uso de interferon B (avonex). Houve, dessa forma, a remissão da quase totalidade dos sintomas e a regressão das lesões visíveis à $\mathrm{RM}$.

Assim, nota-se a relevância da RM como único método diagnóstico in vivo da ECB, visto que quando diagnosticada e tratada precocemente, possibilita que o paciente recupere qualidade em sua vida, através da notável diminuição dos sintomas da doença, apesar de não mudar o prognóstico da doença ${ }^{11}$.

Volpato MG, Klaus DG, Carvalho DC, Gomes NT, Claudino R. Balo's concentric sclerosis case report. Rev Med (São Paulo). 2007 out.-dez.;86(4):207-11.

\begin{abstract}
Goals: The case described forward search to motivate the medical suspicion in front of a probable diagnose of Balo's Concentric Sclerosis (BCS) establishing a routine diagnostic. Methods: Case report with cientific review of articles published about the subject. Results: It is really difficult to determine the BCS's etiology-fisiopathology because of the difuse demyelinating's areas of the central nervous system (CNS) that are seen in a variety of CNS's diseases. Magnetic resonance (MR) is very important to the diagnosis of BCS, but the diagnosis's key is the brain biopsy. The therapy consists of high dose of steroidals being controled by magnetic resonance imaging. Discussion: Once the inflammatory demyelinating nature of the lesion has been established, the difficulty of determination ethiopathology as wide areas of demielynation of the central nervous system can be observed in several neurological diseases. On practice, the BCS's patients show acute or sub-acute symptoms that suggest expansive brain injury. The disease has an unlikely course, that goes to a quickly evolution in weeks or months without remission until a self-limiting that includes several cognitive symptoms like headaches, aphasia, behavioral disturbance, visual dysfunction and ataxia.
\end{abstract}

KEY WORDS: Multiple sclerosis/thiology. Diffuse cerebral sclerosis of schilder/diagnosis. Case reports [Publication type].

\title{
REFERÊNCIAS
}

1. Balo J. Encephalomyelitis periaxialis concentrica. Arch Neurol Psychiatry. 1928;19:242-64.

2. Gonçalves F, Alves S, Cherulli BLB, Mançano AD, Ponte AG. Esclerose concêntrica de Baló: relato de um caso. Rev Bras Radiol Diagn Imagem [online]. Disponível em: URL: http://www.cbr.org.br/cbradiologia2005/ Paineis/08/150/150.htm

3. González-Delgado M, Tuñón A, Guisasola LM, Mateos V. Forma seudotumoral recidivante de esclerosis múltiple. Neurologia. 2004;19(2):83-4.

4. Comi G. Multiple sclerosis: pseudotumoral forms. Neurol Sci 2004;25:S374-S379.

5. Kreindler A, Cardas M, Petrescu A, Macovei M, Rosianu C. Pseudotumoral forms of multiple sclerosis. Rev Roum Neurol. 1973;10(6):569-77.

6. Gu J, Wang R, Lin J, Fang S. Concentric sclerosis: imaging diagnosis and clinical analysis of 3 cases.
Neurol India. 2003;51(4):528-30.

7. Goldman L, Ausiello D. Cecil: Tratado de medicina interna. Rio de Janeiro: Elsevier; 2005.

8. Stadelmann C, Ludwin S, Tabira T, Guseo A, Luchinetti $C F$, Leel-Ossy $L$, et al. Tissue preconditioning may explain concetric lesions in Balo's type of multiple sclerosis. Brain. 2005;128(Pt 5):979-87.

9. Obach V, Gonzalez-Menacho J, Vidal S, Lomeña F, Graus F. TI-201 SPECT in pseudotumoral multiple sclerosis. Clin N Med. 1999;24(3):186-8.

10. Hanemann CO, Kleinschmidt A, Reifenberger G, Freund HJ, Seitz RJ. Balo's concentric sclerosis followed by MRI and positron emission tomography. Neuroradiology. 1993;35(8):578-80.

11. Ng S-H, Ko S-F, Cheung Y-C, Wong H-F, Wan Y-L. MRI features of Balo's concentric sclerosis. Brit J Radiol. 1999;72:400-3. 